\title{
Do Patients with Pancreatic Hyperenzymemia without Abnormal Imaging Need Additional Endoscopic Ultrasound?
}

\author{
Jung Wan Choe and Jong Jin Hyun \\ Division of Gastroenterology, Department of Internal Medicine, Korea University Ansan Hospital, Ansan, Korea
}

See "The Utility of Endoscopic Ultrasound in Patients with Isolated Elevations in Serum Amylase and/or Lipase" by Lalitha M. Sitaraman, Amit H. Sachdev, Tamas A. Gonda, et al., on page 175-181.

The clinical significance of elevated amylase and/or lipase levels in asymptomatic subjects has not been established yet. However, serum amylase and lipase levels are increasingly being examined as part of a routine check-up or during health examination in the absence of symptoms. When pancreatic enzymes are elevated, presence of pancreatitis is first suspected, but if there is no definite evidence of pancreatitis, other pancreatic and extra-pancreatic diseases are considered to explain the elevation of pancreatic enzymes. Other causes that can lead to this condition run the gamut from drugs, anatomical abnormalities of the pancreas, salivary diseases, gastrointestinal diseases, gynecologic diseases, neoplasms, renal failure, macroamylasemia to chronic non-pathological pancreatic hyperenzymemia (CNPH). Imaging tools that can aid in making the diagnosis include abdominal ultrasonography, computed tomography (CT) scan, magnetic resonance cholangiopancreatography (MRCP), MRCP with secretin stimulation (MRCP-S), and endoscopic ultrasound (EUS). The most frequently used imaging modality for diagnosing pancreatitis is CT scan despite its limitations such as inability to identify subtle morphological changes in the pancreas, radiation expo-

Received: February 7, 2019 Accepted: February 8, 2019

Correspondence: Jong Jin Hyun

Division of Gastroenterology, Department of Internal Medicine, Ansan Hospital, Korea University College of Medicine, 123 Jeokgeum-ro, Danwon-gu, Ansan 15355 , Korea

Tel: +82-31-412-5580, Fax: +82-31-8099-6373, E-mail: sean4h@korea.ac.kr ORCID: https://orcid.org/0000-0002-5632-7091

(cc) This is an Open Access article distributed under the terms of the Creative Commons Attribution Non-Commercial License (http://creativecommons.org/ licenses/by-nc/3.0) which permits unrestricted non-commercial use, distribution, and reproduction in any medium, provided the original work is properly cited. sure, and possible occurrence of contrast induced side effects. Endoscopic retrograde cholangiopancreatography (ERCP) is the standard test for diagnosing chronic pancreatitis, which can best demonstrate changes in the pancreatic duct. However, since complications can occur in up to $3 \%-7 \%$ of patients, ERCP is usually performed for therapeutic purposes rather than simply for making diagnosis. Because of the inherent limitations of the aforementioned studies, attempts have been made to detect all possible abnormalities of the pancreas by using tests such as EUS, MRCP, and MRCP-S. MRCP is a noninvasive test that is useful for diagnosing structural abnormalities of the pancreas, and MRCP-S is particularly useful for diagnosing abnormalities of side branch pancreatic duct and delayed main pancreatic duct emptying. ${ }^{1-4}$ EUS is considered to be a highly sensitive test for examining various lesions of the pancreatic duct and parenchyma, and is especially useful for diagnosing chronic pancreatitis compared to other imaging modalities. ${ }^{4,5}$

In the current issue of Clinical Endoscopy, Sitaraman et al. analyzed the usefulness of EUS in patients with isolated elevation of amylase and/or lipase but without pancreatitis or pancreatobiliary disease on noninvasive imaging modalities, such as abdominal ultrasound, CT scan, and MRCP. ${ }^{6}$ This baffling situation has previously been pondered upon by many scientists, and in the 1970s, some researchers like Warshaw and Lee pointed out that excessive examinations should be provided only on the assumption that pancreatic hyperenzymemia was pathologic. ${ }^{7}$ Two decades later in 1996, Gullo reported the result of 18 asymptomatic patients with chronic (>6 months) pancreatic enzyme elevation who were extensively assessed 
but no pancreatic pathology was determined, and defined this condition as $\mathrm{CNPH}^{8}{ }^{8}$ The authors were concerned by that fact that although CNPH patients are more likely to have no specific disease, blood tests and various imaging studies were repeatedly performed and patients' economic burden was increased, leading to deterioration of the quality of life. ${ }^{8}$ Then how should we deal with these patients and what examination should we pursue? Alternatively, it is really necessary even to continue on with additional examination?

There have been multiple studies that looked into the value of MRCP, MRCP-S, and EUS in assessing serum pancreatic enzyme elevation in the absence of definite pancreatic diseases. Studies which assessed the role of MRCP for diagnosing early chronic pancreatitis demonstrated that the sensitivity and specificity were $92 \%$ and $75 \%$, respectively. ${ }^{9,10}$ The incidence of pancreatic cystic lesions found on MRCP was reported to range from $2.4 \%$ to $19.6 \%$, which was not significantly different from the previously reported autopsy data. ${ }^{11}$ Some researchers have emphasized the need for employing MRCP-S because MRCP-S can improve the diagnosis rate of early chronic pancreatitis. ${ }^{12,13}$ MRCP-S has been reported to be able to more clearly confirm the dilatation of the pancreatic duct compared to conventional MRCP. ${ }^{1}$ However, given the fact that additional findings found on MRCP-S are also benign, the clinical significance of MRCP-S is still limited. Other researchers insisted that not only MRCP-S but also EUS should be performed to confirm pancreatic abnormalities. ${ }^{14}$ However, EUS is an expensive and invasive test that is highly dependent on the proficiency of the examiner. Moreover, there are innate limitations to the accuracy of the Rosemont criteria in diagnosing early chronic pancreatitis. ${ }^{15}$ Considering these facts, it is difficult to say that combined EUS \& MRCP-S examinations have superiority and suitability compared to MRCP alone. ${ }^{5}$

Then what value would EUS have when used alone to examine patient with pancreatic hyperenzymemia but without pancreatobiliary diseases on previous imaging studies? In the current study by Sitaraman et al., the authors suggest that EUS is helpful in this subset of patients since it provides a probable diagnosis in $53 \%$ of those with previously normal imaging and contributes to arrive at a diagnosis in $42.8 \%$ even when asymptomatic. ${ }^{6}$ These results are somewhat consistent with those of previous papers. ${ }^{1,14}$ Testoni et al. showed that the percentage of abnormalities found in $\mathrm{CNPH}$ patients and controls using MRCP-S were 52\% and 7.1\%, respectively. ${ }^{1} \mathrm{Di}$ Leo et al. found that the percentage of normal EUS findings in $\mathrm{CNPH}$ group and control group were observed in $39.7 \%$ and $86.8 \%$, respectively. ${ }^{14} \mathrm{CNPH}$ is a diagnosis of exclusion and all pancreas-related diseases must be ruled out before ascertaining that it is truly a benign condition. In this regards, it seems reasonable to conduct additional and aggressive tests to detect pancreatobiliary pathology in those with $\mathrm{CNPH}$ since clinically significant positive findings that can explain elevation of amylase and/or lipase are not infrequently found on MRCP-S and/or EUS.

The study by Sitaraman et al. is a retrospective study with some limitations such as lack of data on alcohol abuse and medication history, and selection bias incurred by selecting only those who underwent EUS examination. ${ }^{6}$ In addition, the study population does not seem to have undergone blood test such as iso-amylase, which could be quite useful in differentiating and ruling out hyperamylasemia of salivary or other origins. Nevertheless, this study can be said to be meaningful in that it refocused the usefulness of EUS in evaluating patients with isolated serum amylase and/or lipase elevations. It remains to be proven which of the two, MRCP-S or EUS, is better in evaluating this condition. EUS might be a good next evaluation step, especially if secretin is not available to carry out MRCP-S as is the situation in Korea. Whichever method is employed, it could particularly be helpful for evaluating those presumed to have $\mathrm{CNPH}$.

\section{Conflicts of Interest}

The authors have no financial conflicts of interest.

\section{REFERENCES}

1. Testoni PA, Mariani A, Curioni S, Giussani A, Masci E. Pancreatic ductal abnormalities documented by secretin-enhanced MRCP in asymptomatic subjects with chronic pancreatic hyperenzymemia. Am J Gastroenterol 2009;104:1780-1786.

2. Donati F, Boraschi P, Gigoni R, et al. Secretin-stimulated MR cholangio-pancreatography in the evaluation of asymptomatic patients with non-specific pancreatic hyperenzymemia. Eur J Radiol 2010;75:e38-e44.

3. Amodio A, Manfredi R, Katsotourchi AM, et al. Prospective evaluation of subjects with chronic asymptomatic pancreatic hyperenzymemia. Am J Gastroenterol 2012;107:1089-1095.

4. Galassi E, Birtolo C, Migliori M, et al. A 5-year experience of benign pancreatic hyperenzymemia. Pancreas 2014;43:874-878.

5. Anaizi A, Hart PA, Conwell DL. Diagnosing chronic pancreatitis. Dig Dis Sci 2017;62:1713-1720.

6. Sitaraman LM, Sachdev AH, Gonda TA, Sethi A, Poneros JM, Gress FG. The utility of endoscopic ultrasound in patients with isolated elevations in serum amylase and/or lipase. Clin Endosc 2019;52:175-181.

7. Warshaw AL, Lee KH. Macroamylasemia and other chronic nonspecific hyperamylasemias: chemical oddities or clinical entities? Am J Surg 1978;135:488-493.

8. Gullo L. Chronic nonpathological hyperamylasemia of pancreatic origin. Gastroenterology 1996;110:1905-1908.

9. Zhang XM, Shi H, Parker L, Dohke M, Holland GA, Mitchell DG. Suspected early or mild chronic pancreatitis: enhancement patterns on gadolinium chelate dynamic MRI. Magnetic resonance imaging. J Magn Reson Imaging 2003;17:86-94.

10. Zhang XM, Mitchell DG, Dohke M, Holland GA, Parker L. Pancreatic cysts: depiction on single-shot fast spin-echo MR images. Radiology 2002;223:547-553.

11. de Jong K, Nio CY, Hermans JJ, et al. High prevalence of pancreatic 
cysts detected by screening magnetic resonance imaging examinations. Clin Gastroenterol Hepatol 2010;8:806-811.

12. Pascual I, Soler J, Peña A, et al. Morphological and functional evaluation of the pancreatic duct with secretin-stimulated magnetic resonance cholangiopancreatography in alcoholic pancreatitis patients. Dig Dis Sci 2008;53:3234-3241.

13. Czakó L. Diagnosis of early-stage chronic pancreatitis by secretin-enhanced magnetic resonance cholangiopancreatography. J Gastroenterol
2007;42 Suppl 17:113-117.

14. Di Leo M, Petrone MC, Zuppardo RA, et al. Pancreatic morpho-functional imaging as a diagnostic approach for chronic asymptomatic pancreatic hyperenzymemia. Dig Liver Dis 2016;48:1330-1335.

15. Catalano MF, Sahai A, Levy M, et al. EUS-based criteria for the diagnosis of chronic pancreatitis: the Rosemont classification. Gastrointest Endosc 2009;69:1251-1261. 